\title{
Eventos de mortalidad accidental de Murciélago rabudo Tadarida teniotis en edificios altos de la ciudad de Valencia (España)
}

\author{
Miguel Ángel Monsalve-Dolz ${ }^{1}$
}

${ }^{1}$ Centros de Recuperación de Fauna-VAERSA-Servicio de Vida Silvestre.
Conselleria de Infraestructuras, Territorio y Medio Ambiente.
CRF "La Granja" de El Saler. Av Los Pinares 106, 46012, El Saler-Valencia.

Corresponding author e-mail: brigada_granja@gva.es

DOI: http://dx.doi.org/10.14709/BarbJ.7.1.2014.07

English title: Accidental mortality events of the European free-tailed bat Tadarida teniotis in high buildings in Valencia (Spain)

\begin{abstract}
Other than events caused by wind turbines, the accidental mortality of European free-tailed bats in Spain has never been reported in the literature. The discovery on five occasions of European free-tailed bats trapped in boxes on a small balcony in the city of Valencia is described here. In two cases, high mortality was recorded. The findings follow a similar pattern: all involve a large number of bats of both sexes, which are found dead in a box with an open top, always in the month of October.
\end{abstract}

Given the number of cases found over the years in a small area and the number of specimens involved, these mortality events could become a determining factor for some colonies.

Keywords: Tadarida teniotis, mortality, boxes, Valencia

Aunque el estatus de conservación del murciélago rabudo (Tadarida teniotis) es “de Preocupación Menor” en el ámbito mundial (Aulagnier et al 2008) en España la información poblacional es escasa, considerándose su población dispersa aunque no rara (Balmori 2007). En la Comunitat Valenciana no se dispone de información sobre la magnitud ni sobre el estado de las poblaciones (Monsalve 2012).

Al igual que el resto de las especies de murciélagos europeos, el murciélago rabudo es una especie longeva por lo que el conocimiento sobre causas de mortalidad es relevante para evaluar y entender su dinámica poblacional. Si los eventos de mortalidad afectan a un número elevado de individuos y se repiten en el tiempo pueden provocar una regresión de las poblaciones. En general son escasos en la literatura los casos de mortalidad accidental en murciélagos (Manville 1963) y más en los que se vean afectados un grupo numeroso de individuos (p.ej. Jung \& Slough 2005). Sobre la especie que nos ocupa no se han encontrado registros documentados, al menos en España, excepto diversos accidentes por choque con aerogeneradores (Camiña 2012, Sánchez et al., 2012).

En este trabajo se describen cinco sucesos similares ocurridos en la ciudad de Valencia en los que un número elevado de murciélagos de esta especie quedan atrapados en diversas estructuras de fachada en edificios altos. En dos de ellos se registra una alta mortalidad. Se discuten las causas que podrían haber causado dichos accidentes.

El primer caso se registró el 5 de octubre de 1995. A raíz de una llamada telefónica avisando del hallazgo de unos murciélagos el personal del centro de recuperación de fauna "La Granja" de El Saler (Valencia) se presentó en una vivienda situada en un $6^{\circ}$ planta -coordenadas geografícas $39^{\circ} 28^{\prime} 40,34^{\prime \prime} \mathrm{N} 0^{\circ} 21^{\prime} 26,41^{\prime \prime} \mathrm{W}$ - y encontraron más de un 


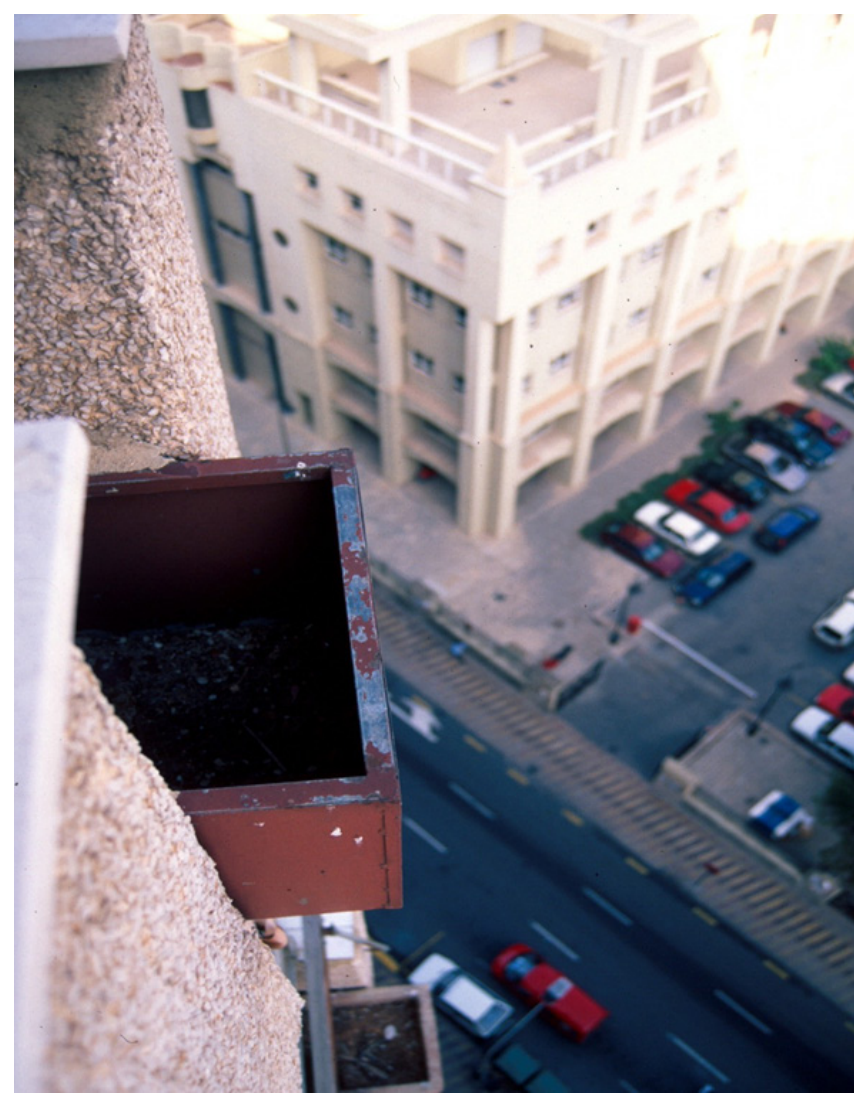

Fig. 1 - Macetero donde fueron encontrados más de 100 murciélagos rabudos muertos en 1996. centenar de ejemplares de murciélagos rabudos atrapados en un pequeño macetero vacío (sin contenido) con forma cúbica, orientado al oeste y con dimensiones 40 × 40 x 40 $\mathrm{cm}$. La persona que dio el aviso había estado escuchando unos chirridos provenientes del exterior de la ventana desde hacía unos días. En el momento de la visita casi todos los ejemplares ya habían muerto y se encontraban en avanzado estado de descomposición. Sólo 19 permanecían vivos, y de ellos 2 estaban muy debilitados y murieron a las pocas horas. Los otros 17 fueron inmediatamente liberados. Dado el escaso espacio existente dentro del macetero la causa de la muerte pudo ser el aplastamiento de unos ejemplares sobre otros.

Un año después, el 8 de octubre de 1996, se registró en el mismo edificio el segundo caso, prácticamente idéntico al anterior. Esta vez se rescataron 2 ejemplares vivos que permanecían junto a más de un centenar muertos en un macetero de las mismas características, situación y orientación que el anterior, pero a una altura de un $10^{\circ}$ piso (ver figura 1). A los pocos días, el 23 de octubre de 1996, se encontraron 43 ejemplares vivos en el mismo macetero donde se registró la mortalidad el año anterior, en la 6 planta. Todos los ejemplares fueron liberados el mismo día.

Estos tres sucesos son muy similares a otro ocurrido en octubre de 2006, a unos $500 \mathrm{~m}$ del edificio donde se registraron los accidentes en 1995 y 1996. Esta vez se trató de 56 murciélagos rabudos que habían quedado atrapados en un macetero de cemento (coordenadas geografícas $\left.39^{\circ} 28^{\prime} 50,75^{\prime \prime} \mathrm{N} 0^{\circ} 21^{\prime} 44,71^{\prime \prime} \mathrm{W}\right)$. Todos los ejemplares fueron

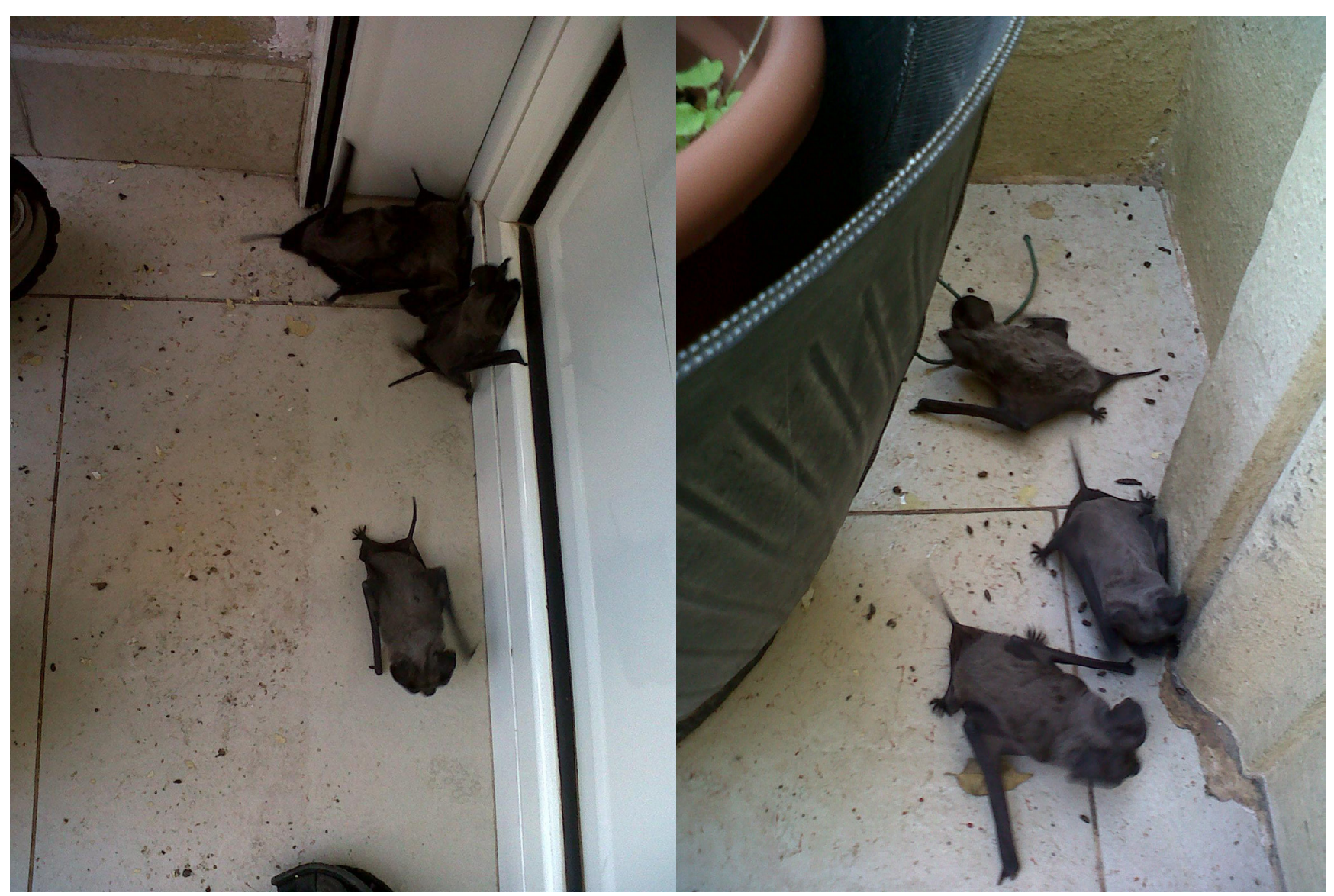

Fig. 2 - Ejemplares encontrados en octubre de 2013 en un balcón de pequeñas dimensiones. 
Tabla. 1 - Fecha, lugar y número de ejemplares afectados en los cinco eventos de mortalidad.

\begin{tabular}{|c|c|c|c|c|c|}
\hline $\begin{array}{l}\text { Fecha } \\
\text { evento }\end{array}$ & $\begin{array}{l}\text { Estructura donde } \\
\text { quedan atrapados }\end{array}$ & $\begin{array}{l}\text { Altura (piso del } \\
\text { edificio) }\end{array}$ & $\begin{array}{l}\mathrm{n}^{\mathrm{o}} \text { ejemplares } \\
\text { encontrados } \\
\text { vivos }\end{array}$ & $\begin{array}{c}\mathrm{n}^{\mathrm{o}} \text { ejemplares } \\
\text { encontrados muertos }\end{array}$ & $\begin{array}{l}\text { sex-ratio (relación } \\
\text { machos/hembras) }\end{array}$ \\
\hline Oct-1995 & macetero & $6^{\mathrm{o}}$ & 19 & $>100$ & - \\
\hline Oct-1996 & macetero & $10^{\circ}$ & 2 & $>100$ & - \\
\hline Oct-1996 & macetero* & $6^{\circ}$ & 43 & 0 & - \\
\hline Oct-2006 & macetero & - & 56 & 0 & $0,81(n=56)$ \\
\hline Oct-2013 & pequeño balcón & $6^{\circ}$ & 56 & 0 & $3,31(\mathrm{n}=56)$ \\
\hline
\end{tabular}

*mismo macetero que en 1995

rescatados tras permanecer allí varios días. En este caso no se registró con exactitud la altura de la planta donde se situaba el macetero pero se trataba de un piso alto. Aunque el tamaño de este macetero era mayor que los primeros con una longitud superior a un metro, su anchura y profundidad fueron similares a los anteriores, aproximadamente unos $40 \mathrm{~cm}$. Todos los ejemplares se sexaron (25 machos y 31 hembras), fueron marcados en antebrazo con anilla homologada y liberados en una zona cercana.

El último caso fue observado el pasado 14 de octubre de 2013. Este día fueron hallados un total de 56 murciélagos rabudos atrapados en un pequeño balcón de un $6^{\circ}$ piso (ver figura 2) en un edificio situado más al sur -coordenadas geografícas 39²7'52,79’'N 0²2’22,18”W-. Las dimensiones del balcón eran de 100 (ancho) x 200 (largo) cm y la altura de la valla de unos $110 \mathrm{~cm}$. En la visita se hallaron un total de 43 machos y 13 hembras. Ningún ejemplar iba marcado con las anillas colocadas en 2006. Todos los ejemplares se encontraban en el suelo del balcón y la mayoría permanecían escondidos en los únicos refugios disponibles, dentro de unas zapatillas de deporte y detrás de una maceta. Al parecer la barandilla del balcón con paredes de cemento lisas representaba una barrera infranqueable que los murciélagos no podían superar.

En los dos primeros casos descritos probablemente se tardó más en avisar al centro de recuperación de fauna; ese factor pudo provocar que la cantidad de ejemplares afectados fuera mayor y se encontraran ejemplares con buen aspecto general junto a otros con síntomas de inanición y ejemplares muertos ya en descomposición. En ambos se registró alta mortalidad de ejemplares (Tabla 1). Por el contrario, a partir del tercer caso, con menos ejemplares afectados, el tiempo

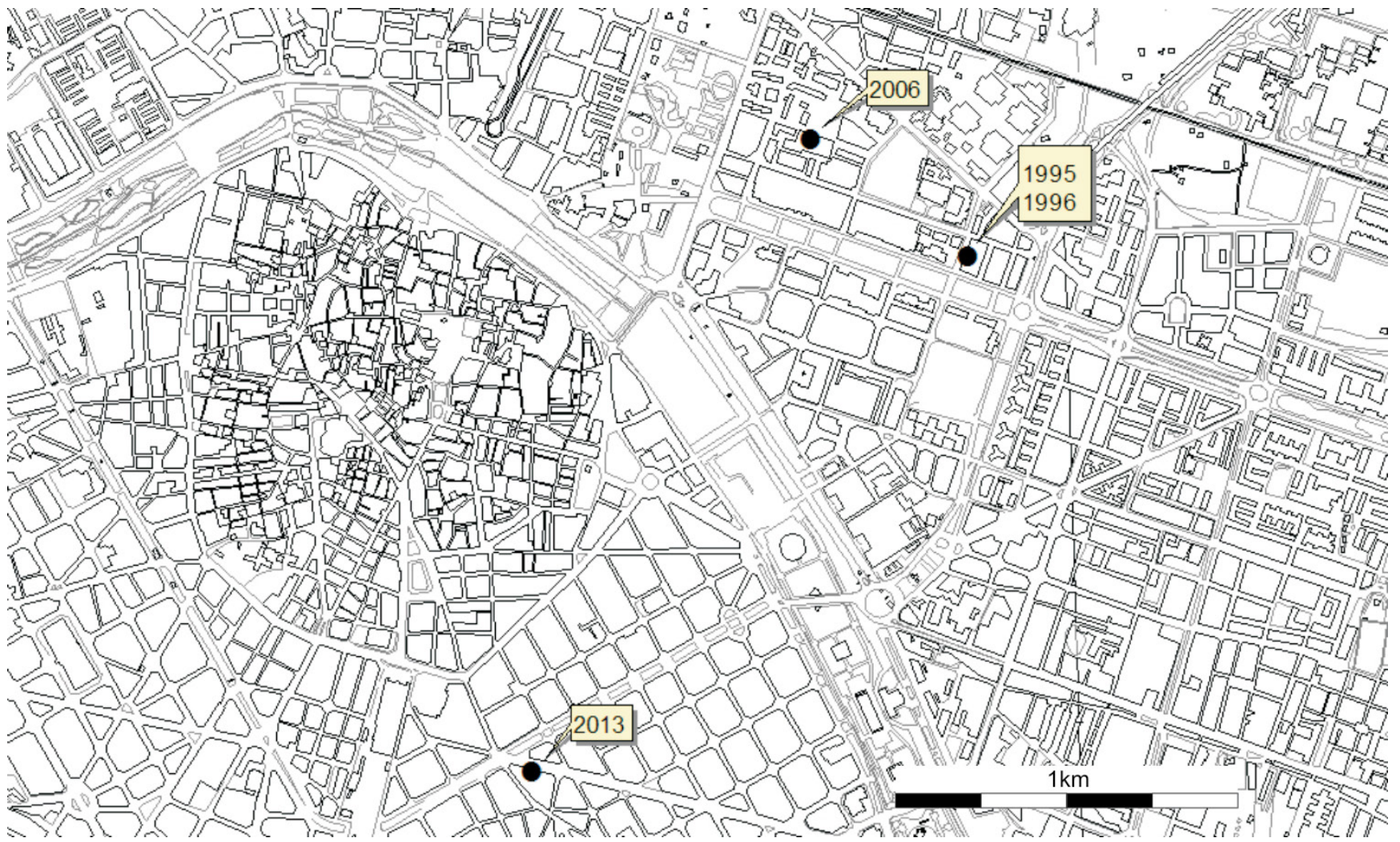

Fig. 3 - Localización de los lugares donde se encontraron los murciélagos accidentados en la ciudad de Valencia. Se señala el año de los hallazgos. 
transcurrido desde el primer ejemplar accidentado hasta el rescate fue menor (estimado entre 4 y 5 días).

Todos los hallazgos siguen un patrón similar, se ven afectados varias decenas de murciélagos, aparecen numerosos ejemplares de ambos sexos, quedan atrapados en la fachada de un edificio a gran altura, el lugar donde quedan atrapados es una estructura abierta por la parte superior pero sin salida en suelo y paredes, y por último, todos los sucesos se han producido en el mes de octubre.

Al parecer en todos los sucesos los murciélagos fueron quedando atrapados de forma progresiva, seguramente durante varias noches. La persona que se encontró los murciélagos accidentados en 2013 observó como cada día había algunos más que el anterior. Por otra parte, en ningún caso después de la retirada de los ejemplares atrapados hubo nuevos ejemplares accidentados, lo que apunta a que los accidentes estuvieron relacionados con algún comportamiento social que incluía atracción de unos ejemplares hacia otros. Esto viene reforzado por el hecho de que en el momento de la liberación de los murciélagos rescatados alguno de ellos realizó vuelos bajos aproximándose a pocos centímetros de la caja donde permanecía el resto de los individuos, los cuales emitían fuertes sonidos sociales desde el interior de la misma.

La época en la que se producen los accidentes coincide con el celo de la especie, cuando los machos y hembras se agrupan formando harenes, sin embargo las agrupaciones en esta época suelen ser pequeñas. El número máximo de ejemplares controlados en dos refugios de Valladolid a lo largo de tres temporadas fue de 17 (Balmori 2003). El tamaño de las agrupaciones descritas en el presente artículo son mayores y, aunque siempre se observan machos y hembras, la sex-ratio fue diferente en los dos eventos en los que se registró.

En el mismo estudio se observó una agrupación de juveniles muy ruidosa en uno de los refugios durante el mes de octubre (Balmori 2003). En los casos aquí descritos no se vio ningún ejemplar con el característico pelaje claro de los juveniles, no obstante en una exploración de los ejemplares encontrados en 2013 tampoco se observó que los machos presentaran testículos desarrollados, por lo que no hay que descartar la posibilidad de que se trate de individuos jóvenes, nacidos en la primavera, cuya inexperiencia podría explicar, al menos en parte, estos eventos de mortalidad.

Dado el número de casos encontrados a lo largo de los años en un área limitada y la cantidad de ejemplares afectados podría tratarse de un factor de mortalidad determinante para algunas colonias. Sería recomendable recoger mayor información en el futuro y comprobar cómo se producen estos accidentes.

\section{Agradecimientos}

David Almenar y Cati Gerique me acompañaron en algunas visitas realizadas a los edificios afectados. Además, Cati Gerique fue la veterinaria encargada del CRF La Granja cuando ocurrieron los primeros accidentes. Ella supervisó y determinó la causa de muerte de los cadáveres hallados en 1995 y 1996.

\section{REFERENCIAS}

Aulagnier, S., Paunovic, M., Karatas, A., Palmeirim, J., Hutson, A.M., Spitzenberger, F., Juste, J. \& BendA, P. 2008. Tadarida teniotis. En: UICN 2013. Lista Roja de Especies Amenazadas. Versión 2013.2. < www. iucnredlist.org $>$.

BALMORI, A. 2003. Avances en el conocimiento de la biología y organización social del murciélago rabudo (Tadarida teniotis). Galemys 15 (n'especial), 2003. Pp: 37-53.

BAlmori A. 2007 Tadarida teniotis (Rafinesque, 1814) Pp: 267-271. En: L.J. Palomo, J. Gisbert \& J.C. Blanco (eds.). Atlas y Libro Rojo de los Mamíferos Terrestres de España. Dirección General para la Biodiversidad. SECEM-SECEMU, Madrid.

CAmiña A. 2012. Bat Fatalities at Wind Farms in Northern Spain - Lessons to be Learned. Acta Chiropterologica 14(1):205-212. DOI: http://dx.doi. org/10.3161/150811012X654402

Jung, T.S., \& Slough B.G. 2005. Mortality of Little Brown Bats, Myotis lucifugus, in a rodent trap in the boreal forest. Canadian Field-Naturalist 119(4): 589-590.

Manville, R. H. 1963. Accidental mortality in bats. Mammalia. 27: 361-366. DOI: https://dx.doi. org//10.1515/mamm.1963.27.3.361

Monsalve M. A. 2012. Tadarida teniotis Pp: 248-250. En: Jiménez J., Monsalve M.A. \& Raga J.A. (Eds) 2012. Mamíferos de la Comunitat Valenciana. Colección Biodiversidad, 19. Conselleria d'Infraestructures, Territori i Medi Ambient. Generalitat Valenciana, Valencia.

SÁnchez S, Berrío-Martínez, J. \& IBÁÑEZ, C. 2012. Patrones de mortalidad de murciélagos en Parques Eólicos del Sur de la Península Ibérica. Póster, I Congreso Ibérico sobre Energía Eólica y Conservación de la Fauna, Jerez, Cádiz, enero 2012. 